\title{
Raman-dressed spin-1 spin-orbit-coupled quantum gas
}

\author{
Zhihao Lan ${ }^{1, *}$ and Patrik Öhberg ${ }^{2}$ \\ ${ }^{1}$ Mathematical Sciences, University of Southampton, Highfield, Southampton SO17 1BJ, United Kingdom \\ ${ }^{2}$ SUPA, Institute of Photonics and Quantum Sciences, Heriot-Watt University, Edinburgh EH14 4AS, United Kingdom
}

(Received 7 November 2013; published 24 February 2014)

\begin{abstract}
The recently realized spin-orbit-coupled quantum gases [Lin et al., Nature (London) 471, 83 (2011); Wang et al., Phys. Rev. Lett. 109, 095301 (2012); Cheuk et al., ibid. 109, 095302 (2012)] mark a breakthrough in the cold atom community. In these experiments, two hyperfine states are selected from a hyperfine manifold to mimic a pseudospin-1/2 spin-orbit-coupled system by the method of Raman dressing, which is applicable to both bosonic and fermionic gases. In this paper, we show that the method used in these experiments can be generalized to create any large pseudospin spin-orbit-coupled gas if more hyperfine states are coupled equally by the Raman lasers. As an example, we study, in detail, a quantum gas with three hyperfine states coupled by the Raman lasers and show, when the state-dependent energy shifts of the three states are comparable, triple-degenerate minima will appear at the bottom of the band dispersions, thus, realizing a spin-1 spin-orbit-coupled quantum gas. A novel feature of this three-minima regime is that there can be two different kinds of stripe phases with different wavelengths, which has an interesting connection to the ferromagnetic and polar phases of spin-1 spinor Bose-Einstein condensates without spin-orbit coupling.
\end{abstract}

DOI: 10.1103/PhysRevA.89.023630

\section{INTRODUCTION}

Cold atoms have proven to be ideal platforms to simulate a variety of phenomena ranging from condensed matter to nuclear physics due to their unprecedented controllability [1,2]. Among these, a great amount of theoretical and experimental efforts in recent years have been dedicated to the engineering of gauge potentials for neutral atoms $[3,4]$. Not only because it provides a platform to simulate magnetic fields and spin-orbit couplings (a special form of non-Abelian gauge potentials) and related phenomena, such as quantum spin Hall effects or topological phases [5-7] in condensed-matter physics, but also due to the dramatic impact the gauge potentials have on the system dynamics. For instance, spin-orbit effects with bosons have no counterpart in the electronic properties of solids. Even at the single-particle level, the introduction of gauge potentials will modify the particle dispersions, leading to exotic properties, such as negative reflection [8] or multirefringence $[9,10]$. These modified particle dispersions will have dramatic effects on the few-body or many-body physics when interactions are present. Indeed, the enhanced density of states by the gauge potentials leads to two-body bound states even at the Bardeen-Cooper-Schrieffer (BCS) side of the resonance [11]. Moreover, the broken Galilean invariance due to the presence of the gauge potentials could result in finite-momentum Cooper pairs [12]. As a consequence, the possibility of Bose-Einstein condensates (BECs) to $\mathrm{BCS}$ crossover by increasing the strength of the gauge coupling [13-15] or the possible realization of the long-sought Fulde-Ferrell-Larkin-Ovchinnikov superfluidity $[16,17]$ at the many-body level has attracted great interest.

To date, synthetic spin-orbit couplings have been realized experimentally for both BEC and degenerate Fermi gases [4]. The key idea behind these achievements, i.e., Raman dressing, was first demonstrated in a series of experiments by Lin and co-workers [18-21] for a ${ }^{87} \mathrm{Rb} \mathrm{BEC}$ and later on for fermionic

\footnotetext{
*z.lan@ soton.ac.uk
}

PACS number(s): 03.75.Hh, 05.30.Jp, 67.85.-d, 71.70.Ej

${ }^{40} \mathrm{~K}$ in Ref. [22] and with ${ }^{6} \mathrm{Li}$ in Ref. [23]. The elegance of this method is in its simplicity where only one pair of lasers and an external magnetic field are used. Both the Abelian and the non-Abelian regimes can be reached by tuning the laser power. For example, in the first three experiments at the National Institute of Standards and Technology (NIST), only a single minimum of the lowest energy dispersion in the form of $\hbar^{2}\left(k_{x}-A_{x}\right)^{2} / 2 m$ was created where a constant $A_{x}$, space dependent $A_{x}$, and time dependent $A_{x}$ lead to a uniform vector potential with zero magnetic field, nonzero magnetic field $B_{z}=-\partial_{y} A_{x} \neq 0$, and nonzero electric field $E_{x}=-\partial_{t} A_{x} \neq$ 0 , respectively. Conversely, two minima in the energy dispersion are interpreted as two dressed spin states responsible for the synthetic spin-orbit coupling with equal Rashba and Dresselhaus strengths. We note that the most recent experimental [24,25] and theoretical [26-29] studies using this Raman scheme are mostly concerned with the two-minima regime. It is, however, not an insurmountable task to experimentally control all three Zeeman levels [30] and, by doing so, to obtain a spin-1 scenario. Magnetically generated spin-orbit coupling [31] may also provide a viable route to larger spin systems.

In this paper, we show that a three-minima regime in the energy dispersion of the NIST setup can be reached. We show, in detail, how this three-minima regime can emerge as a function of the Raman strength $\Omega_{R}$ and the quadratic Zeeman energy $\varepsilon$ when the detuning $\delta$ is zero and the contributions of the three Zeeman states of the underlying manifold are comparable. This is in contrast to the extensively studied phase diagram in the $\Omega_{R}-\delta$ plane [21,24,25]. For a special configuration of the parameters, a triple-degenerate minima can appear at the bottom of the spectrum, thus, realizing a spin-1 spin-orbit-coupled quantum gas. Our paper shows that the method of Raman dressing can readily be used to synthesize large pseudospin-orbit couplings for neutral atoms if more hyperfine states are coupled equally.

\section{THE THREE-MINIMA REGIME}

We follow the NIST setup shown in Fig. 1(a) where two counterpropagating Raman lasers along $\hat{x}$ with frequency 

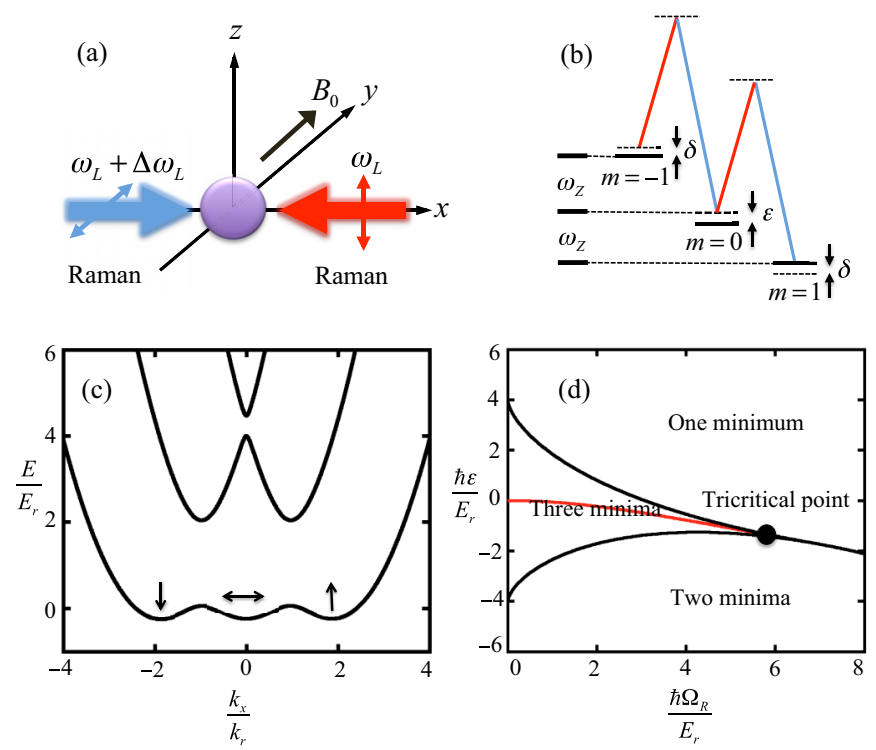

FIG. 1. (Color online) (a) Schematic of the experimental setup at NIST. Two counterpropagating Raman lasers with frequencies $\omega_{L}$ and $\omega_{L}+\Delta \omega_{L}$ along $\hat{x}$ impinge on the atomic cloud. A bias field $B_{0}$ along $\hat{y}$ produces the Zeeman effects. (b) Level diagram of the Raman coupling scheme within the $F=1$ manifold. The linear and quadratic Zeeman shifts are $\omega_{Z}$ and $\varepsilon$ while detuning from Raman resonance $\delta$, which is set to zero in this study. Atoms excited by the Raman lasers will change their spin projection along the magnetic field by 1 while increasing their linear momentum by $2 \hbar k_{r}$. (c) The spectrum of $\mathscr{H}\left(\tilde{k}_{x}\right)$ with $\hbar \Omega_{R}=2 E_{r}$ and $\hbar \varepsilon=-0.23 E_{r}$. The triple-degenerate minima at the bottom of the spectrum serves as a spin-1 system. (d) The single-particle phase diagram in the plane of $\Omega_{R}-\varepsilon$. The three phases meet at a tricritical point, and the red (grey) line shows the regime where the three minima are degenerate in energy.

difference $\Delta \omega_{L}$ and momentum difference $2 \hbar k_{r}$ couple the three hyperfine states of an ultracold atomic cloud. At the single-particle level, the setup is applicable to both bosonic and fermionic gases as long as suitable hyperfine states can be selected. For simplicity, we will denote the three states as an $F=1$ manifold $\left|F, m_{F}\right\rangle=|1,-1\rangle,|1,0\rangle,|1,+1\rangle$, although, in principle, they can be three hyperfine states of a much higher manifold. Meanwhile, there is a magnetic field along $\hat{y}$ producing the linear and quadratic Zeeman shifts $\hbar \omega_{Z}$ and $\hbar \varepsilon$ for the three states. The lasers induce a Raman transition in the atom, transferring linear momentum $2 \hbar k_{r} \hat{x}$ to the atom while increasing its spin angular momentum by $\hbar$ at the same time [Fig. 1(b)]. The Hamiltonian of the system, dressed by the Raman lasers, is described by $\mathscr{H}=\hbar^{2} k^{2} / 2 m-\hbar \Omega_{R} / 2\left[e^{i\left(2 q_{r} x-\Delta \omega_{L} t\right)}\left(\mathscr{F}_{z}+i \mathscr{F}_{x}\right)+\right.$ H.c. $]-$ $\hbar \omega_{Z} \mathscr{F}_{y}+\hbar \varepsilon \mathscr{F}_{y}^{2}$, where $\mathscr{F}$ is the spin-1 operator, $\Omega_{R}$ is the Raman frequency associated with the Raman process, and $\hbar \omega_{Z}$ and $\hbar \varepsilon$ are the linear and quadratic Zeeman energies of the three levels. In the rotating-wave approximation for the frame in spin space rotating about $\hat{y}$ with frequency $\Delta \omega_{L}$, the Hamiltonian becomes static $\mathscr{H}=\hbar^{2} k^{2} / 2 m-$ $\hbar \Omega_{R}\left[\cos \left(2 q_{r} x\right) \mathscr{F}_{z}-\sin \left(2 q_{r} x\right) \mathscr{F}_{x}\right]-\hbar\left(\omega_{Z}-\Delta \omega_{L}\right) \mathscr{F}_{y}+$ $\hbar \varepsilon \mathscr{F}_{y}^{2}$. We can then apply another rotation in spin space along $\hat{y}$ with angle $2 \hbar k_{r}$, and the Hamiltonian in the state basis of $\Psi\left(\tilde{k}_{x}\right)=\left\{\left|-1, \tilde{k}_{x}-2 k_{r}\right\rangle,\left|0, \tilde{k}_{x}\right\rangle,\left|1, \tilde{k}_{x}+2 k_{r}\right\rangle\right\}$, labeled by the wave vector of quasimomentum $\tilde{k}_{x}$, reduces to $\mathscr{H}=\mathscr{H}\left(\tilde{k}_{x}\right)+\left[\hbar^{2}\left(k_{y}^{2}+k_{z}^{2}\right) / 2 m\right]$ with $\mathscr{H}\left(\tilde{k}_{x}\right)$ for the Raman coupling given by [18]

$$
\mathscr{H}\left(\tilde{k}_{x}\right)=\hbar\left(\begin{array}{ccc}
\frac{\hbar\left(\tilde{k}_{x}+2 k_{r}\right)^{2}}{2 m}-\delta & \Omega_{R} / 2 & 0 \\
\Omega_{R} / 2 & \frac{\hbar \tilde{k}_{x}^{2}}{2 m}-\hbar \varepsilon & \Omega_{R} / 2 \\
0 & \Omega_{R} / 2 & \frac{\hbar\left(\tilde{k}_{x}-2 k_{r}\right)^{2}}{2 m}+\delta
\end{array}\right),
$$

where $\delta=\left(\Delta \omega_{L}-\omega_{Z}\right)$ is the detuning from Raman resonance. It is to be noted that parameters $\delta$ and $\varepsilon$ just shift the three bare branches up and down. Although the experiments $[21,24,25]$ use $\delta$ to select two out of the three Zeeman states as a spin-1/2 system, here we set $\delta=0$ and leave only $\varepsilon$ as a free parameter to balance the contributions of the three bare branches, which in principle, can be controlled by state-dependent trapping potentials, i.e., both positive and negative $\varepsilon$ 's can be realized in this way. Alternatively, negative quadratic Zeeman energy can also be realized experimentally by the technique of microwave dressing (e.g., see Ref. [32]). In the following, we define $E_{r}=\hbar^{2} k_{r}^{2} / 2 m$ as the recoil energy which will be used as the energy scale and $k_{r}$ as the momentum scale.

Figure 1(d) shows the single-particle phase diagram of Hamiltonian (1) in the plane of Raman coupling $\left(\Omega_{R}\right)$ and energy shift of the middle branch $(\varepsilon)$. The three phases, characterized by one minimum, two minima, and three minima in the lowest energy dispersion, meet at a tricritical point beyond which the three-minima regime no long exists. The red line in Fig. 1(d) shows the regime where the three minima are exactly degenerate in energy. In Fig. 1(c), we show one example of the triple-degenerate-minima regime with parameters of $\hbar \Omega_{R}=2 E_{r}$ and $\hbar \varepsilon=-0.23 E_{r}$. In this case, the three-degenerate minima at the bottom of the spectrum serve as a spin-1 manifold, and the atomic gas is spin-orbit coupled with an enlarged pseudospin of 1 . We note our phase diagram in the plane of $\Omega_{R}-\varepsilon$ is very different from the phase diagram in the plane of $\Omega_{R}-\delta$ (e.g., see Ref. [24]). The phase diagram in the plane of $\Omega_{R}-\delta$ shows only two phases, a two-local-minima regime or a one-minimum regime, and when the Raman coupling is strong enough, only one single minimum can exist. We also note tricriticality and similar phase diagrams in spin-orbit-coupled BEC were discussed recently by Li et al. [28] but in a two-minima regime at a many-particle level, which is different from our three-minima regime at the single-particle level.

The physical reason for the phase diagram in Fig. 1(d) can be understood as follows. At $\left(\Omega_{R}, \varepsilon\right)=(0,0)$, the energy dispersions are the three bare parabolas located at $\tilde{k}_{x}^{\mathrm{min}} / k_{r}=$ $-2,0,2$. With increasing $\Omega_{R}$, gaps will open at the anticrossing points, and $\varepsilon$ will shift the middle branch up with negative $\varepsilon$ or down with positive $\varepsilon$. When the middle branch shifts up (with a decreasing $\varepsilon$ ), the minimum located at $\tilde{k}_{x}=0$ will merge with the two neighboring maxima into a single maximum, thus, the system enters the two-minima regime. Conversely, when the middle branch shifts down (with increasing $\varepsilon$ ), the two minima located at $\tilde{k}_{x}^{\min } / k_{r}=-2,2$ will merge with its neighboring maximum and will leave only a minimum at $\tilde{k}_{x}=0$, thus, the system enters the single-minimum regime. When the Raman 
coupling is strong enough, the minimum located at $\tilde{k}_{x}=0$ will be destroyed by the anticrossing between the dispersion curves, and as a result, the three-minima regime no longer exists.

\section{MOMENTUM-RESOLVED RADIO-FREQUENCY SPECTROSCOPY}

By using the same method of Raman dressing as in the NIST experiments, spin-orbit-coupled Fermi gases have also been realized experimentally at ShanXi University $\left({ }^{40} \mathrm{~K}\right)$ [22] and the Massachusetts Institute of Technology $\left({ }^{6} \mathrm{Li}\right)$ [23] where the band dispersions have been studied by momentum-resolved radio-frequency (rf) spectroscopy and spin-injection spectroscopy, respectively. Although the spininjection spectroscopy uses a rf laser to inject free atoms in a reservoir state into the empty spin-orbit-coupled system, after which the momentum and spin of injected atoms are mapped out by using time-of-flight and spin-resolved detections, the momentum-resolved rf spectroscopy uses a rf laser to transfer atoms from one of the hyperfine states for constructing the spin-orbit-coupling system to an empty reservoir state. For a noninteracting system, the momentum-resolved rf spectroscopy yields equivalent information to the spin-injection spectroscopy.

We have calculated the momentum-resolved rf spectroscopy in order to show experimentally it is possible to observe the three-minima band structure shown in Fig. 1(c) (see Ref. [33] for details of the calculation and Ref. [34] for a recent experiment). We present the results in Fig. 2 where plots (a)-(c) show the momentum-resolved rf spectroscopy for the three Zeeman states that are selected to synthesize the spin-orbit coupling and (d) shows the reconstructed band structure by the combination of plots (a)-(c). The reason for reconstructing the band dispersions in Fig. 1(c) by using the rf spectroscopies of all the three Zeeman states is because each branch of the band dispersions is a mixture of the bare dispersions of the three Zeeman states. From Fig. 2(d), we see the qualitative features of the band structure in Fig. 1(c) are clearly visible. The rf spectrum also shows an important feature of the system, i.e., the weights of the three dressed spin states are largely determined by the three bare branches, which is the essential point for the emerging of two different stripe phases to be discussed later.

\section{STRIPE PHASES FROM THE THREE MINIMA}

There are two possible phases for the BEC in the two-minima regime when interactions are present, a planewave phase and a stripe phase depending on whether a single minimum or two minima are occupied [26]. For our three-minima case, at the single-particle level, the ground state for a BEC is triple degenerate and is described by $\Phi_{m}(x)=A_{+} \chi_{m}^{p_{+}}(x)+A_{0} \chi_{m}^{p_{0}}(x)+A_{-} \chi_{m}^{p_{-}}(x)$, where $A_{ \pm, 0}$ is the complex amplitude and $\chi_{m}^{p_{ \pm}, 0}(x)=e^{i p_{ \pm, 0} x} \tilde{\chi}_{m}^{p_{ \pm, 0}}$ with $\tilde{\chi}_{m}^{p_{ \pm}, 0}$ as the spinor from the lowest eigenstate of the single-particle Hamiltonian (1) at the three minima of $k_{x}= \pm k_{0}, 0$. At the many-particle level, the interaction will select which minimum or minima the system will condensate to by minimizing the interaction energy. For example, a single nonzero component

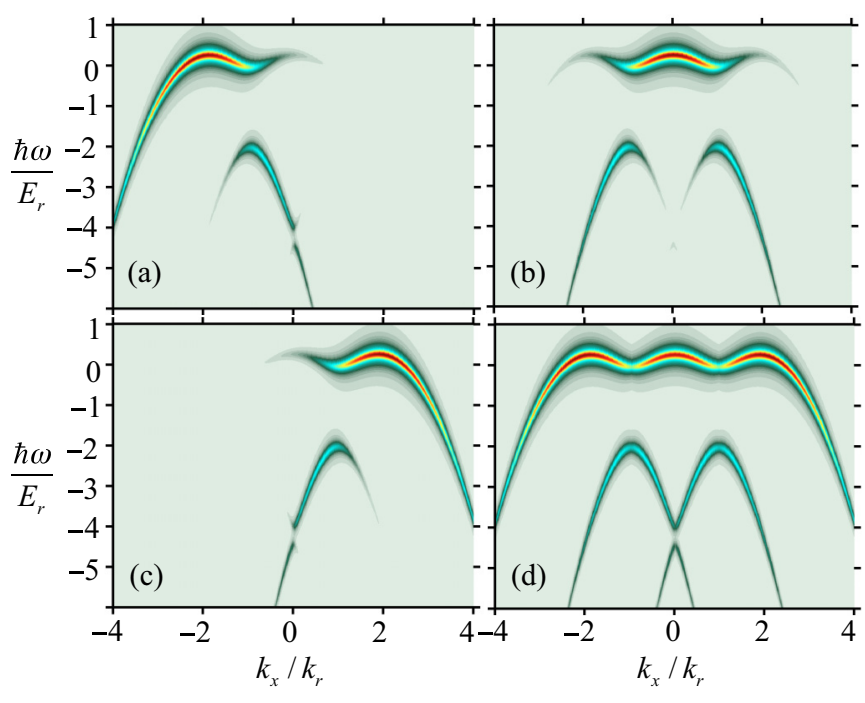

FIG. 2. (Color online) Momentum-resolved rf spectroscopy for reconstructing the band dispersions in Fig. 1(c) for a spin-orbitcoupled Fermi gas. Plots (a)-(c) show momentum-resolved rf spectroscopies for the three Zeeman states, respectively, whereas, (d) shows the reconstructed band dispersions by the combination of (a)-(c). Parameters used: $\hbar \Omega_{R}=2 E_{r}, \hbar \varepsilon=-0.23 E_{r}$, chemical potential $\mu=3 E_{r}$, and temperature $T=0.6 \mu$ and in consideration of the energy resolution of the spectroscopy $\gamma \sim 0.1 E_{r}$. We have replaced the $\delta$ function for the energy conservation by $\delta(x)=(\gamma / \pi) /\left[x^{2}+\right.$ $\left.\gamma^{2}\right][23,33]$. Note, when increasing the chemical potential, the transfer strength will become stronger, and the higher branches will also get occupied since there will be more and more atoms in the system.

of $A_{ \pm, 0}$ means a plane-wave phase, whereas, two nonzero components of $A_{ \pm, 0}$ create a standing-wave phase [26,35]. One interesting consequence of the triple-degenerate-minima regime is that there are two different kinds of stripe phases with different wavelengths. When the BEC occupies the two minima at $k_{x}= \pm k_{0}$, the resulting stripe phase has twice the smaller wavelength than when the BEC occupies the two minima at $k_{x}=k_{0}$ and $k_{x}=0$ or $k_{x}=-k_{0}$ and $k_{x}=0$.

The interaction Hamiltonian for a three-component BEC is given by $\hat{\mathscr{H}}_{\text {int }}=\int d^{3} r g_{0} \hat{n}^{2}(r)+g_{2} \hat{\mathscr{F}}^{2}(r)[35,36]$, where $\hat{n}=\sum_{m} \hat{n}_{m}$ is the total population of the three Zeeman states and $\hat{\mathscr{F}}=\phi_{\alpha} \mathscr{F}_{\alpha \beta} \phi_{\beta}$ is the spin-1 operator with $\mathscr{F}$ as the spin-1 generalization of the Pauli matrix. The complex amplitudes $A_{ \pm, 0}$ are determined by minimizing the GrossPitaevski functional of the single-particle Hamiltonian plus the interaction Hamiltonian. In the numerical investigations, apart from the plane-wave phase as discussed before [26,28,35], which results from the occupation of a single minimum, we also find two kinds of stripe phases with different wavelengths. Figure 3 shows a typical example for the density of the $m=0$ Zeeman state at two different $g_{0}$ 's and $g_{2}$ 's where the difference of factor 2 in wavelength is clearly seen. Note that, since these two kinds of stripe phases have the same laser parameters, i.e., they stem from the same single-particle dispersion, the change in wavelength by the laser parameter as discussed in Ref. [26] cannot explain their origin.

To better understand the nature of the two different stripe phases, we write the density of one of the Zeeman states 
(a)

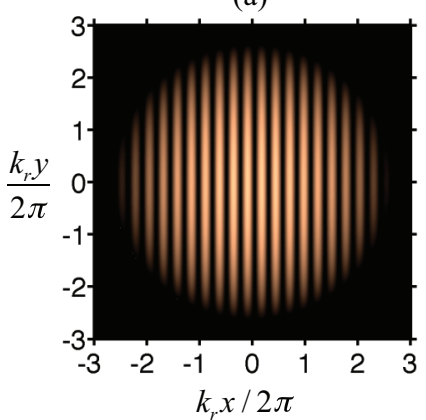

(b)

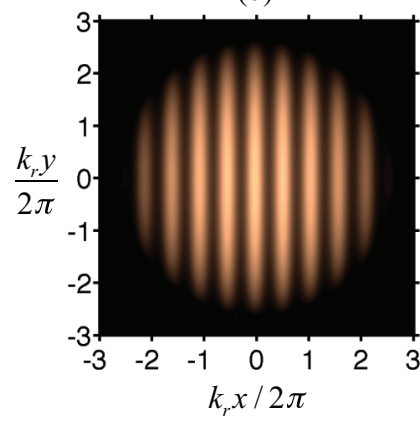

FIG. 3. (Color online) Two typically different kinds of stripe phases from the three-minima band structure in Fig. 1(c) for a BEC with $k_{0}=1.88 k_{r}$ when collisional interactions are present. Shown are the density distributions of the $m=0$ Zeeman state with (a) $g_{0} / g_{2}=1$ and (b) $g_{0} / g_{2}=-1$, where $g_{0}>0$ for both cases. (A harmonic trap is taken account for in the numerics by the local density approximation.) The typical parameters realized experimentally as in Ref. [21] are $E_{r} / \hbar=1.1 \times 10^{4} \mathrm{~Hz}, g_{0}=7.79 \times 10^{-12} \mathrm{~Hz} \mathrm{~cm}^{3}$, and the number of atoms $N=1.8 \times 10^{5}$.

(e.g., $m=0)$ as $n_{0}=\phi_{0} \phi_{0}^{*}$ with $\phi_{0}=\left(A_{-} e^{-i k_{0} x} a_{-}+A_{0} a_{0}+\right.$ $A_{+} e^{i k_{0} x} a_{+}$), where $a_{ \pm, 0}$ (taken as real) is the $m=0$ component of the spinor wave function and $A_{ \pm, 0}$ is the complex amplitude. Because of the nonzero overlap between the spinor part of the wave function, the density of each Zeeman state will develop a stripe structure. A straightforward calculation gives $n_{0}=C+$ $\left(C_{1} e^{i k_{0} x}+\right.$ c.c. $)+\left(C_{2} e^{2 i k_{0} x}+\right.$ c.c. $)$, where $C=\left|A_{-}\right|^{2}\left|a_{-}\right|^{2}+$ $\left|A_{0}\right|^{2}\left|a_{0}\right|^{2}+\left|A_{+}\right|^{2}\left|a_{+}\right|^{2}, C_{1}=a_{0}\left(A_{-}^{*} a_{-} A_{0}+A_{+} a_{+} A_{0}^{*}\right)$, and $C_{2}=A_{-}^{*} a_{-} A_{+} a_{+}$. For $g_{0}>0$, we find that, when $g_{0} / g_{2}=1$, the values of $A_{ \pm, 0}$, which minimize the interaction energy, always have vanishing $A_{0}$, which means $C_{1}=0$, thus, the wavelength of the stripe phase is $\pi / k_{0}$ in this case. For $g_{0} / g_{2}=-1$, the $A_{ \pm, 0}$ 's are all nonvanishing. But in this case, since $a_{0}$ is dominant [see, for instance, Fig. 2(b)], the term $e^{i k_{0} x}$ is dominant over $e^{2 i k_{0} x}$, which means the wavelength of the stripe in this case is given by $2 \pi / k_{0}$. The two different stripe phases, therefore, originate from the separation between the three minima. A further physical insight may be gained by the fact that the weights of the three dressed branches are largely determined by the three Zeeman states themselves (e.g., see Fig. 2), i.e., the minimum at $\pm k_{0}, 0$ by the Zeeman state $m=\mp 1,0$, respectively. So when $g_{2}>0\left(g_{2}<0\right)$, the system wants a zero (large) spin to minimize (maximize) the interaction energy, consequently, $m= \pm 1(m=1,0$ or $m=-1,0)$ are occupied. This shows the two different kinds of stripe phases have an interesting

connection with the ferromagnetic and polar phases of spin-1 BEC [36], which is the true manifestation of spin-orbit coupling in this system, i.e., the structure in pseudospin space (ferromagnetic or polar) has been transferred to structures in orbit space (small or large wavelength stripe) since the three dressed spin states are represented by the three minima with different momenta. It is certainly tempting to conjecture that other types of stripe phases will emerge when including more Zeeman states. Since the two different kinds of stripe phases originate from the sign of $g_{2}$ when the $g_{2}$ term is dominating over the $g_{0}$ term (assumed positive), we could tune $g_{0} / g_{2}$ from +1 to -1 to see the transition of the wavelength from $\pi / k_{0}$ to $2 \pi / k_{0}$. The dynamics of the transition would depend on the experimental details, such as, for instance, nonadiabatic effects. The interaction parameters for observing these stripe phases can be reached experimentally by optical Feshbach resonance [37] (see also a recent experiment [38] for Ramaninduced Feshbach resonance in this setup), and the different stripe structures can be probed by Bragg light scattering [39] or can be detected by measuring the displacement of the atomic cloud after expansion when the trap is turned off [26].

\section{CONCLUSIONS AND DISCUSSIONS}

Motivated by the recent experiments on synthetic spinorbit-coupled quantum gases [21-25] in the two-minima regime, we investigated and showed how a three-minima regime in this setup can be obtained. We found that, when the contributions of the three Zeeman states are comparable, triple-degenerate minima appear at the bottom of the band dispersions, which can be translated into a spin-orbit-coupled spin-1 quantum gas. We further found there are two different kinds of stripe phases in this setup which have their roots from the ferromagnetic and polar phases of spin-1 BEC, i.e., due to spin-orbit coupling, the structure in pseudospin space is manifested by the structure in orbit space. The scenario can be generalized to create a spin-orbit-coupled high spin quantum gas by including more Zeeman states. We note that, recently, a different experimental technique to create two minima in momentum space by shaking an optical lattice and in situ observation of ferromagnetic domains has been achieved [40]. These techniques could also be applied to the three-minima regime studied in this paper.

\section{ACKNOWLEDGMENTS}

We would like to thank Y.-J. Lin and I. Spielman for helpful comments and suggestions. Z.L. acknowledges support from EPSRC Grant No. EP/I018514/1, and P.Ö. acknowledges support from EPSRC Grant No. EP/J001392/1.
[1] M. Lewenstein, A. Sanpera, V. Ahufinger, B. Damski, A. S. De, and U. Sen, Adv. Phys. 56, 243 (2007).

[2] I. Bloch, J. Dalibard, and S. Nascimbène, Nat. Phys. 8, 267 (2012).

[3] J. Dalibard, F. Gerbier, G. Juzeliunas, and P. Öhberg, Rev. Mod. Phys. 83, 1523 (2011).
[4] V. Galitski and I. B. Spielman, Nature (London) 494, 49 (2013).

[5] M. Z. Hasan and C. L. Kane, Rev. Mod. Phys. 82, 3045 (2010).

[6] N. Goldman, I. Satija, P. Nikolic, A. Bermudez, M. A. MartinDelgado, M. Lewenstein, and I. B. Spielman, Phys. Rev. Lett. 105, 255302 (2010); N. Goldman, J. Beugnon, and F. Gerbier, ibid. 108, 255303 (2012). 
[7] M. C. Beeler, R. A. Williams, K. Jiménez-García, L. J. LeBlanc, A. R. Perry, and I. B. Spielman, Nature (London) 498, 201 (2013).

[8] G. Juzeliunas, J. Ruseckas, A. Jacob, L. Santos, and P. Öhberg, Phys. Rev. Lett. 100, 200405 (2008).

[9] Z. Lan, N. Goldman, A. Bermudez, W. Lu, and P. Öhberg, Phys. Rev. B 84, 165115 (2011); Z. Lan, A. Celi, W. Lu, P. Öhberg, and M. Lewenstein, Phys. Rev. Lett. 107, 253001 (2011).

[10] M. P. Kennett, N. Komeilizadeh, K. Kaveh, and P. M. Smith, Phys. Rev. A 83, 053636 (2011); B. Roy, P. M. Smith, and M. P. Kennett, Phys. Rev. B 85, 235119 (2012).

[11] J. P. Vyasanakere and V. B. Shenoy, Phys. Rev. B 83, 094515 (2011).

[12] L. Dong, L. Jiang, H. Hu, and H. Pu, Phys. Rev. A 87, 043616 (2013).

[13] M. Gong, S. Tewari, and C. Zhang, Phys. Rev. Lett. 107, 195303 (2011).

[14] H. Hu, L. Jiang, X.-J. Liu, and H. Pu, Phys. Rev. Lett. 107, 195304 (2011).

[15] Z.-Q. Yu and H. Zhai, Phys. Rev. Lett. 107, 195305 (2011).

[16] Z. Zheng, M. Gong, X. Zou, C. Zhang, and G. Guo, Phys. Rev. A 87, 031602(R) (2013).

[17] F. Wu, G. C. Guo, W. Zhang, and W. Yi, Phys. Rev. Lett. 110, 110401 (2013).

[18] Y.-J. Lin, R. L. Compton, A. R. Perry, W. D. Phillips, J. V. Porto, and I. B. Spielman, Phys. Rev. Lett. 102, 130401 (2009).

[19] Y.-J. Lin, R. L. Compton, K. Jiménez-García, J. V. Porto, and I. B. Spielman, Nature (London) 462, 628 (2009).

[20] Y.-J. Lin, R. L. Compton, K. Jiménez-García, W. D. Phillips, J. V. Porto, and I. B. Spielman, Nat. Phys. 7, 531 (2011).

[21] Y.-J. Lin, K. Jiménez-García, and I. B. Spielman, Nature (London) 471, 83 (2011).

[22] P. Wang, Z.-Q. Yu, Z. Fu, J. Miao, L. Huang, S. Chai, H. Zhai, and J. Zhang, Phys. Rev. Lett. 109, 095301 (2012).
[23] L. W. Cheuk, A. T. Sommer, Z. Hadzibabic, T. Yefsah, W. S. Bakr, and M. W. Zwierlein, Phys. Rev. Lett. 109, 095302 (2012).

[24] J.-Y. Zhang, S.-C. Ji, Z. Chen, L. Zhang, Z.-D. Du, B. Yan, G.-S. Pan, B. Zhao, Y. J. Deng, H. Zhai, S. Chen, and J.-W. Pan, Phys. Rev. Lett. 109, 115301 (2012).

[25] C. Qu, C. Hamner, M. Gong, C. Zhang, and P. Engels, Phys. Rev. A 88, 021604(R) (2013).

[26] T.-L. Ho and S. Zhang, Phys. Rev. Lett. 107, 150403 (2011).

[27] S. Sinha, R. Nath, and L. Santos, Phys. Rev. Lett. 107, 270401 (2011).

[28] Y. Li, L. P. Pitaevskii, and S. Stringari, Phys. Rev. Lett. 108, 225301 (2012).

[29] Z. Chen and H. Zhai, Phys. Rev. A 86, 041604 (2012).

[30] I. Spielman (private communication).

[31] B. M. Anderson, I. B. Spielman, and G. Juzeliūnas, Phys. Rev. Lett. 111, 125301 (2013).

[32] F. Gerbier, A. Widera, S. Fölling, O. Mandel, and I. Bloch, Phys. Rev. A 73, 041602(R) (2006).

[33] X.-J. Liu, Phys. Rev. A 86, 033613 (2012).

[34] Z. Fu, L. Huang, Z. Meng, P. Wang, X.-J. Liu, H. Pu, H. Hu, and J. Zhang, Phys. Rev. A 87, 053619 (2013).

[35] C. Wang, C. Gao, C.-M. Jian, and H. Zhai, Phys. Rev. Lett. 105, 160403 (2010).

[36] T.-L. Ho, Phys. Rev. Lett. 81, 742 (1998); T. Ohmi and K. Machida, J. Phys. Soc. Jpn. 67, 1822 (1998).

[37] C. Chin, R. Grimm, P. Julienne, and E. Tiesinga, Rev. Mod. Phys. 82, 1225 (2010).

[38] R. A. Williams, M. C. Beeler, L. J. LeBlanc, K. Jiménez-García, and I. B. Spielman, Phys. Rev. Lett. 111, 095301 (2013).

[39] H. Miyake, G. A. Siviloglou, G. Puentes, D. E. Pritchard, W. Ketterle, and D. M. Weld, Phys. Rev. Lett. 107, 175302 (2011).

[40] C. V. Parker, L.-C. Ha, and C. Chin, Nat. Phys. 9, 769 (2013). 